Michel Monsigny

Patrick Midoux Annie-Claude Roche

\section{ADRESSE}

M. Monsigny: professeur de biochimie. P. Midoux: chargé de recherche à l'Inserm. A.-C. Roche : directeur de recherche à l'Inserm. Département de biochimic des glycoconjugućs et lectines endogènes, centre de biophysique moléculaire, Cnrs et universitć d'Orléans, 1, rue Haute, 45071 Orlćans Cedex, France.

\title{
Perspectives ex vivo et in vivo, pour la thérapie génique, de la transfection sélective à l'aide de complexes plasmide-polylysine ciblés
}

Transférer de l'ADN dans des cellules, ex vivo et in vivo, efficacement et en toute innocuité est le rêve de toutes les équipes cherchant à mettre au point des méthodes de thérapie génique. A ce titre, les méthodes utilisant des transporteurs inertes, non viraux, comporteront toujours un préjugé favorable par rapport aux vecteurs viraux si leur efficacité peut être démontrée, car ils posent nettement moins de problèmes potentiels de sécurité. La polylysine, polycation formant des complexes avec l'ADN, pourrait être la base de telles méthodes. En effet, son couplage à différents agents, notamment des sucres reconnus par des lectines particulières, permet $d$ 'en cibler l'effet vers des cellules et des tissus donnés. De plus, son association à des substances bloquant la dégradation lysosomiale et favorisant le passage dans le cytosol des complexes contenant l'ADN permet d'atteindre, d'ores et déjà, de très bonnes efficacités de transfert dans différents systèmes.

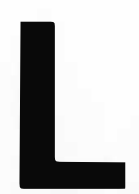

e ciblage sélectif des agents thérapeutiques est un vieux rêve qui hante l'esprit de nombreux chercheurs et thérapeutes depuis P. Ehrlich au début du siècle, et qui semble maintenant être à portée de main. En effet, les vingt dernières années ont été marquées par des progrès significatifs dans la connaissance des récepteurs présents à la surface des cellules et dans leur capacité à entrấner leur ligand à l'intérieur de la cellule. En particulier, à la fin des années 1960, G. Ashwell, A. Morell et leurs collègues découvrent que cer- taines glycoprotéines ayant leur copule glucidique partiellement dégradée par perte de résidus acide-Nacétyl neuraminique (asialoglycoprotéines) sont très rapidement et très efficacement éliminées de la circulation sanguine. Les mêmes auteurs établissent par la suite le mécanisme moléculaire : les asialoglycoprotéines sont reconnues par une lectine membranaire des cellules du parenchyme hépatique et sont convoyées à l'intérieur de vésicules vers les lysosomes [1]. La lectine membranaire reconnaît des structures glucidiques comportant un résidu galactose en position termi- 


\section{RÉFÉRENCES}

1. Ashwell G, Harford J. Carbohydratespecific reccptors of the liver. Annu Rev Biochem $1982 ; 51: 531-54$.

2. Monsigny $\mathrm{M}$, Roche AC, Kieda C, Midoux P, Obrenovitch A. Characterization and biological implications of membranc lectins in tumor, lymphoïd and mycloïd cells. Biochimie 1988 ; 70 : 1633-49.

3. Sharon $\mathbf{N}$, Lis $\mathrm{H}$. Lectin as cell recognition molccules. Science $1989 ; 246: 227-33$.

4. Varki A. Selectin and other mammalian sialic acid-binding lectins. Curr Op Cell Biol $1992 ; 4: 257-66$.

5. Brown MS, Anderson RGW, Goldstcin JL. Recycling receptors: the round trip itinerary of migrant membrane proteins. Cell $1983 ; 32$ : 663-7.

6. Dautry-Varsat A. Reccptor mediated endocytosis: the intraccllular journcy of transf errin and its receptor. Biochimie 1986 ; 68 : 375-81.

7. Posner BI, Patel B, Verna AK, Bergeron JJM. Uptake of insulin by plasmalleme and Golgi subcellular fraction of rat liver. $J$ Biol Chem 1980 ; 255 : 735-41.

8. Louvard D. Mécanismes moléculaires du trafic intracellulaire : sćcrétion et endocytose par réccptcur. médecine/sciences 1988 ; 4 (suppl au $\mathrm{n}^{\circ} 10$ ) : 6-20

9. Monsigny $\mathrm{M}$, Roche $\mathrm{AC}, \mathrm{Midoux} \mathrm{P}$. Endogenous lectins and drug targeting. Ann NY Acad Sci 1988 ; 551 : 399-414.

10. Fiume L, Corrado B, Matioli A, Spinosa G. Targeting of antiviral drugs bound to protein carriers. Crit Rev Ther Drug Carrier Syst $1988 ; 4$ : 265-84.

11. Duncan R. Drug-polymer conjugates potential for improved chemotherapy. Anticancer Drugs 1992; 3 : 175-220.

12. Bonfils E, Depierreux C, Midoux $P$, Thuong NT, Monsigny M, Roche AC Drug targeting: synthesis and endocytosis of oligonucleotide-ncoglycoprotein conjugates. Nucleic Acids Res 1992 ; 20 : 4621-9.

13. Bonfils E, Mendès C, Roche AC, Monsigny $M$, Midoux $P$. Uptake by macrophages of a biotinylated oligo- $\alpha$-deoxythymidylate by using mannosylated streptavidin. Bioconjugate Chem $1992 ; 3$ : 277-84.

14. Backer JM, Kahn CR, White MF. The dissociation and degradation of internalized insulin occur in the endosomes of rat hepatoma cells. $J$ Biol Chem 1990; 265 : 14828-35.

15. White J, Kiclian M, Helenius A. Membranc fusion proteins of enveloped animal nale, c'est-à-dire un galactose ayant toutes ses fonctions alcooliques libres, ce qui est le cas de glycoprotéines sériques désialylées [1]. Depuis, il a été établi que de nombreuses cellules animales possèdent des lectines membranaires [2-4] qui reconnaissent spécifiquement des osides de structures diverses.

Parallèlement, de nombreuses autres protéines membranaires capables de fixer et d'entraîner leur ligand à l'intérieur de la cellule ont été décrites. C'est le cas, en particulier, des récepteurs des lipoprotéines [5], de la transferrine [6], de l'insuline [7] et de divers autres facteurs de croissance, cytokines, modulateurs, etc. [8].

Tirant partie des propriétés des protéines membranaires agissant comme récepteurs et capables d'introduire efficacement leur ligand dans le cytoplasme, une nouvelle approche thérapeutique a été développée au cours des dix dernières années en utilisant des ligands (naturels, modifiés ou synthétiques) comme transporteurs d'agents antitumoraux, antiviraux, antiparasitaires, ou d'immunomodulateurs [9-11].

Plus récemment, ce même concept a été étendu au transport sélectif d'oligonucléotides [12, 13] et, de façon remarquable, au transfert de gènes, c'est-à-dire à la transfection de cellules en culture et même à la transfection de cellules in vivo.

\section{Comment faire pénétrer un gène dans le cytosol et/ou le noyau?}

Les techniques habituellement utilisées pour introduire un gène dans des cellules en culture ne sont pas spécifiques, c'est-à-dire qu'elles ne sont pas fondées sur des mécanismes de reconnaissance: c'est le cas, par exemple, de l'électroporation, du déchirement mécanique ou osmotique de la membrane plasmique, des techniques utilisant le précipité de phosphate de calcium, le diéthylaminoéthyl dextran (DEAE dextran), des lipides cationiques (DOTMA, DAPMA, DOTAP, etc.) ou des liposomes. En outre, ces techniques ne sont pas ou ne sont que difficilement transposables à la transfection in vivo. En revanche, les techniques utilisant des virus modifiés sont sélectives des cellules possédant le récepteur du virus. En effet, ce n'est qu'après reconnaissance des glycoprotéines de la membrane virale par un composé de la membrane de la cellule cible que les membranes virale et cellulaire fusionnent. Cette fusion se fait soit à la surface de la cellule, donc à $\mathrm{pH}$ neutre (c'est le cas, par exemple, des paramyxovirus), soit dans des endosomes intracellulaires (comme dans le cas des orthomyxovirus et des rétrovirus).

Le transport des plasmides par des macromolécules susceptibles d'être reconnues spécifiquement par des composés de la membrane plasmique des cellules cibles relève d'une démarche imitant le mécanisme d'entrée du matériel génétique viral dans la cellule. Dans tous les cas décrits jusqu'à présent, le complexe plasmidetransporteur macromoléculaire est reconnu spécifiquement par un récepteur membranaire qui entraîne le complexe dans des vésicules d'endocytose, dans des endosomes, et probablement dans d'autres compartiments intracellulaires plus profonds, plus éloignés de la membrane plasmique (figure 1).

La membrane plasmique s'invagine et entraîne, dans un puits qui se referme sur lui-même en donnant une vésicule, le ligand en interaction avec le récepteur membranaire. Le contenu de la vésicule ainsi formée est à $\mathrm{pH}$ neutre, celui du milieu environnant, mais très rapidement il devient acide. Les vésicules et leur contenu fusionnent, soit entre elles, soit avec d'autres compartiments membranaires. Dans le cas du transport de la transferrine [6], le récepteur et son ligand (la transferrine) sont rapidement recyclés vers la surface. Dans le cas du transport des asialoglycoprotéines [1], les vésicules contenant le récepteur et l'asialoglycoprotéine pénètrent plus profondément, mais le récepteur, qui perd son affinité pour l'asialoglycoprotéine dès que le $\mathrm{pH}$ devient acide, est finalement recyclé vers la membrane plasmique, alors que l'asialoglycoprotéine libre dans la lumière d'une vésicule est transférée dans des lysosomes où elle sera dégradée. Dans le cas de l'insuline [14], l'hormone se dissocie également de son récepteur dans les endosomes acides ; pour une part, elle retourne 
à la surface, où elle est libérée, pour une autre part, elle est dégradée dans les endosomes. Des fragments d'insuline radioactifs ont également été retrouvés dans l'appareil de Golgi [7], dans le cytosol [14] et vraisemblablement dans le noyau (figure 2).

Le trafic intracellulaire et le devenir des molécules internalisées à l'aide des récepteurs peuvent être modulés par divers agents tels que la chloroquine, la monensine, la bréfeldine $\mathrm{A}$, etc.

La chloroquine (figure 3) est une amine lysosomotrope ; elle s'accumule dans les compartiments acides et, de ce fait, ralentit leur acidification avec une double conséquence : i) ralentissement de la dissociation récepteurligand qui intervient normalement en milieu acide, et ii) ralentissement de l'hydrolyse du matériel endocyté, en particulier de la protéolyse. En outre, la présence de chloroquine ralentit également le passage des ligands des compartiments endocytiques aux lysosomes.
La monensine est un ionophore qui, en échangeant les ions $\mathrm{H}^{+}$et $\mathrm{Na}^{+}$à travers les membranes, neutralise les compartiments acides. De ce fait, la monensine inhibe les deux processus qui ont lieu normalement à $\mathrm{pH}$ acide et lors de la fusion des vésicules.

L'efficacité du transfert de gène dépendra de la nature du transporteur et du chemin qu'il est susceptible d'emprunter à l'intérieur de la cellule. L'efficacité du passage du gène d'un compartiment endocytique au cytosol pourra être augmentée par addition de composés tels que la chloroquine, qui peut prolonger le séjour du gène dans des compartiments où le passage à travers la membrane peut avoir lieu et ralentir le transfert dans les lysosomes, et donc ralentir les processus de dégradation. L'efficacité du transfert de gène pourra également être augmentée par des artifices, tels que l'addition de peptides fusiogènes. On sait qu'une glycoprotéine membranaire des orthomyxovirus, par exemple, possède une

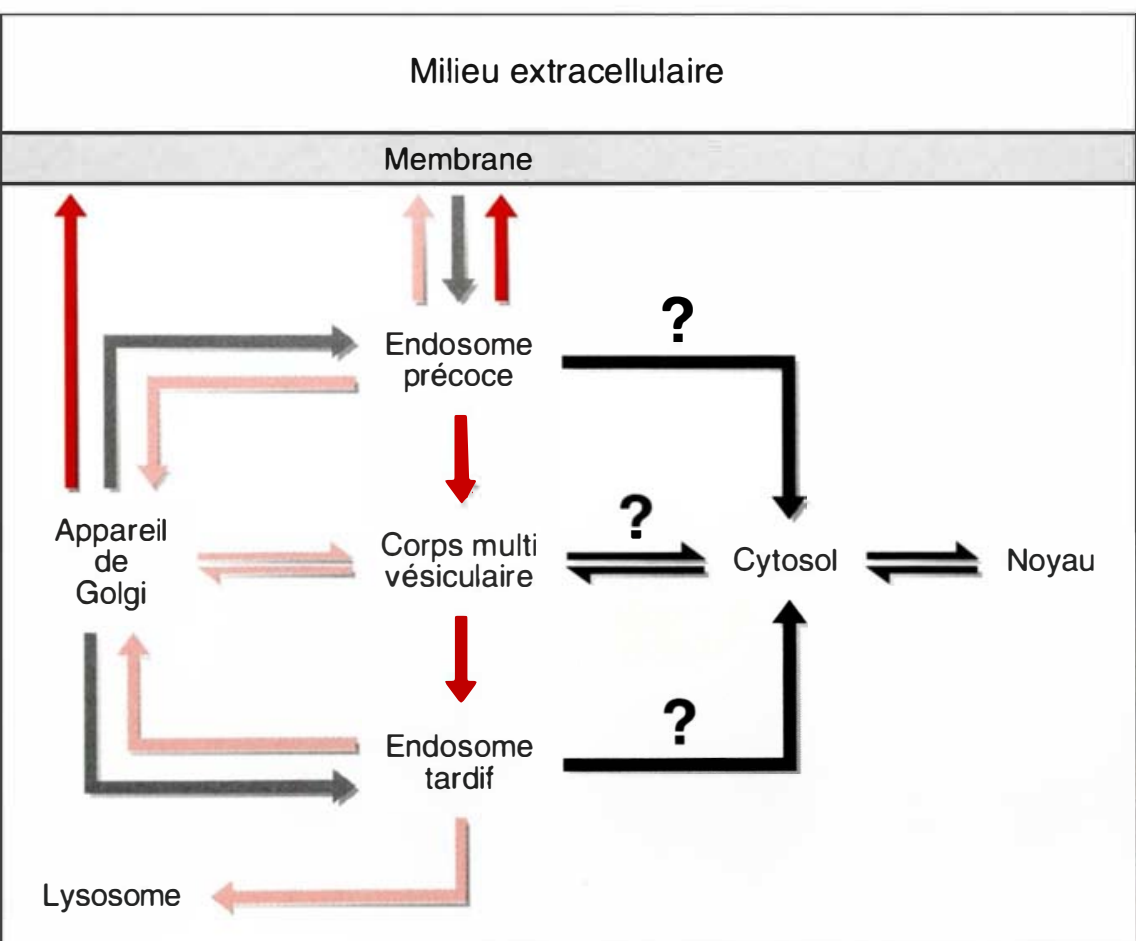

Figure 1. Schéma des chemins intracellulaires de macromolécules endocytées. Les flèches grises, roses et rouges correspondent au transport vésiculaire; les flèches noires correspondent au trafic des macromolécules libres. Les flèches grises correspondent aux vésicules mantelées, les flèches rouges aux vésicules lisses, les flèches roses à un transport vésiculaire et/ou à un transfert dû à des prolongements tubulaires transitoires.

$\mathrm{m} / \mathrm{s} \mathrm{n}^{\circ} 4 \mathrm{vol} .9$, avril 93 séquence $\mathrm{N}$-terminale qui, en milieu acide, induit la fusion entre la membrane virale et la membrane d'un endosome [15]. Les peptides fusiogènes dérivant de cette séquence $\mathrm{N}$ terminale peuvent favoriser le passage du gène dans le cytosol en favorisant la fusion des membranes, en particulier dans un compartiment endosomique appelé compartiment multivésiculaire qui apparaît en microscopie électronique comme une large vésicule contenant des vésicules plus petites [16].

\section{Formation d'un complexe entre un plasmide et un polycation lié à une asialoglycoprotéine}

Les acides nucléiques sont des macromolécules polyanioniques qui - en tant que telles - interagissent de façon coopérative avec des polycations. Parmi les polycations les plus connus, les polyamines sont des composés naturels associés aux acides nucléiques dans les cellules. Des polycations synthétiques, tels que le DEAE dextran et la polylysine, qui sont utilisés pour transfecter des cellules, forment des complexes avec les acides nucléiques.

En 1987, Wu et Wu [17] ont eu l'idée de lier la polylysine à une asialoglycoprotéine : l'asialo-orosomucoïde ou ASOR qui, depuis les travaux d'Ashwell et de Morell, est connue pour être un excellent ligand de la lectine membranaire des cellules du parenchyme hépatique (figure 4). La poly-L-lysine et l'ASOR ont été liés de façon covalente par formation de ponts disulfures après introduction de résidus pyridinyl dithiopropanoyle sur chacun des partenaires (SPDP) (figure 5). La réaction de couplage se fait en milieu dénaturant ( $2 \mathrm{M}$ chlorure de guanidinium) de haute force ionique, compte tenu de la faible solubilité de l'ASOR et de la relative incompatibilité entre la poly-L-lysine, qui est un polymère polycationique, et l'ASOR, qui est une glycoprotéine de caractère amphi-ionique. Le conjugué obtenu est ensuite mélangé avec un plasmide, en l'occurrence le pSV2-CAT, qui contient le gène de la chloramphénicol acétyl transférase sous la dépendance du promoteur précoce et du enhancer du virus SV40. 


\section{RÉFÉRENCES}

16. Hopkins CR. Intraccllular routing of transferrin and transferrin receptors in cpidermoid carcinoma cells. Cell 1983 ; 34 : 321-30.

17. Wu GY, Wu CH. Receptor-mediated in vitro gene transformation by a soluble DNA carricr system. I Biol Chem 1987 ; 262 : 4429-32.

18. Wu GY, Wu CH. Evidence for targeted gene delivery to HepG2 hepatoma cells in vitro. Biochemistry 1988 ; 27 : 887-92.

19. Wu CH, Wilson JM, Wu GY. Targcting genes : delivery and persistent expression of a forcign gene driven by mammalian regulatory clements in vivo. J Biol Chem $1989 ; 264: 16985-7$

20. Wu GY, Wilson JM, Shalaby F, Grossman M, Shafritz DA, Wu CH. Reccptormediated gene delivery in vivo. Partial correction of genetic analbuminemia in Nagase rats. J Biol Chem 1991; 266 : 14338-42.

21. Wilson JM, Grossman M, Wu CH, Chowdhury NR, Wu GY, Chowdhury JR. Hepatocyte-directed gene transfer in vivo leads to transient improvement of hypercholesterolemia in low density lipoprotein receptor deficient rabbits. J Biol Chem 1992 ; $267: 963-7$.

22. Huckett B, Ariatti M, Hawtrcy AO Evidence for targeted gene transfer by receptor mediated endocytosis. Stable expression following insulin directed entry of neo into HepG2 cclls. Biochem Pharmacol 1990 ; 40 : 253-63

23. Rosenkranz AA, Yachmencv SV, Jans DA, et al. Receptor-mediated cndocytosis and nuclear transport of a transfecting DNA construct. Exp Cell Res 1992 ; 199 : 323-9.

24. Wagner E, Zenke M, Cotten M, Beug H, Birnsticl ML. Transferrin-polycation conjugate as carricrs for DNA uptake into cclls. Proc Natl Acad Sci USA $1990 ; 87$ : 3410-4.

25. Zenke $\mathrm{M}$, Stcinlein $\mathrm{P}$, Wagner $\mathrm{E}$, Cotten M, Bcug H, Birnsticl MI. Receptor-mediated endocytosis of transferrin polycation conjugates : an efficient way to introduce DNA into hematopoictic cells. Proc Natl Acad Sci USA 1990 ; 87 : 3655-9.

26. Cotten M, Langle-Rouault F, Kirlappos $\mathrm{H}$, et al. Transferrin-polycation-mediated introduction of DNA into human lcukemic cells : stimulation by agents that affect the survival of transfected DNA or modulate transf crrin receptor levels. Proc Natl Acad Sci USA 1990 ; 87 : 4033-7.

27. Cotten M, Wagner E, Zatloukal K, Phillips S, Curicl DT, Birnsticl ML. Highefficiency receptor-mediated delivery of small and large (48 kilobase) gene constructs using the endosome-disruption activity of defective or chemically inactivated adenovirus particles. Proc Natl Acad Sci USA 1992 ; 89 : 6094-8.
Ce complexe permet un transfert suffisamment efficace dans des cellules en culture exprimant la lectine spécifique des asialoglycoprotéines (la cellule HepG2, un hépatome humain) pour démontrer que l'enzyme transfectée est exprimée sous forme active. La transfection est sélective puisque, avec diverses autres cellules en culture, qui n'expriment pas la lectine spécifique des asialoglycoprotéines, l'activité chloramphénicol acétyl transférase n'a pas pu être détectée [18]. Ce procédé de transport sélectif est également efficace in vivo, puisqu'après injection par voie intraveineuse d'un plasmide complexé au conjugué ASOR- polylysine, la protéine correspondant au gène transfecté est exprimée transitoircment après 24 heures, et si l'on pratique une hépatectomie partielle 30 minutes après l'injection du complexe, la protéine transfectée est encore exprimée 11 semaines plus tard [19]. Sur cette base, des stratégies de thérapie génique peuvent être envisagées. Les premiers exemples concernent unc correction partielle de l'analbuminémie chez les rats Nagase [20] et une réduction transitoire de l'hypercholestérolémie par transfert du récepteur de la lipoprotéine de faible densité (LDL-R) à des lapins génétiquement déficients [21].

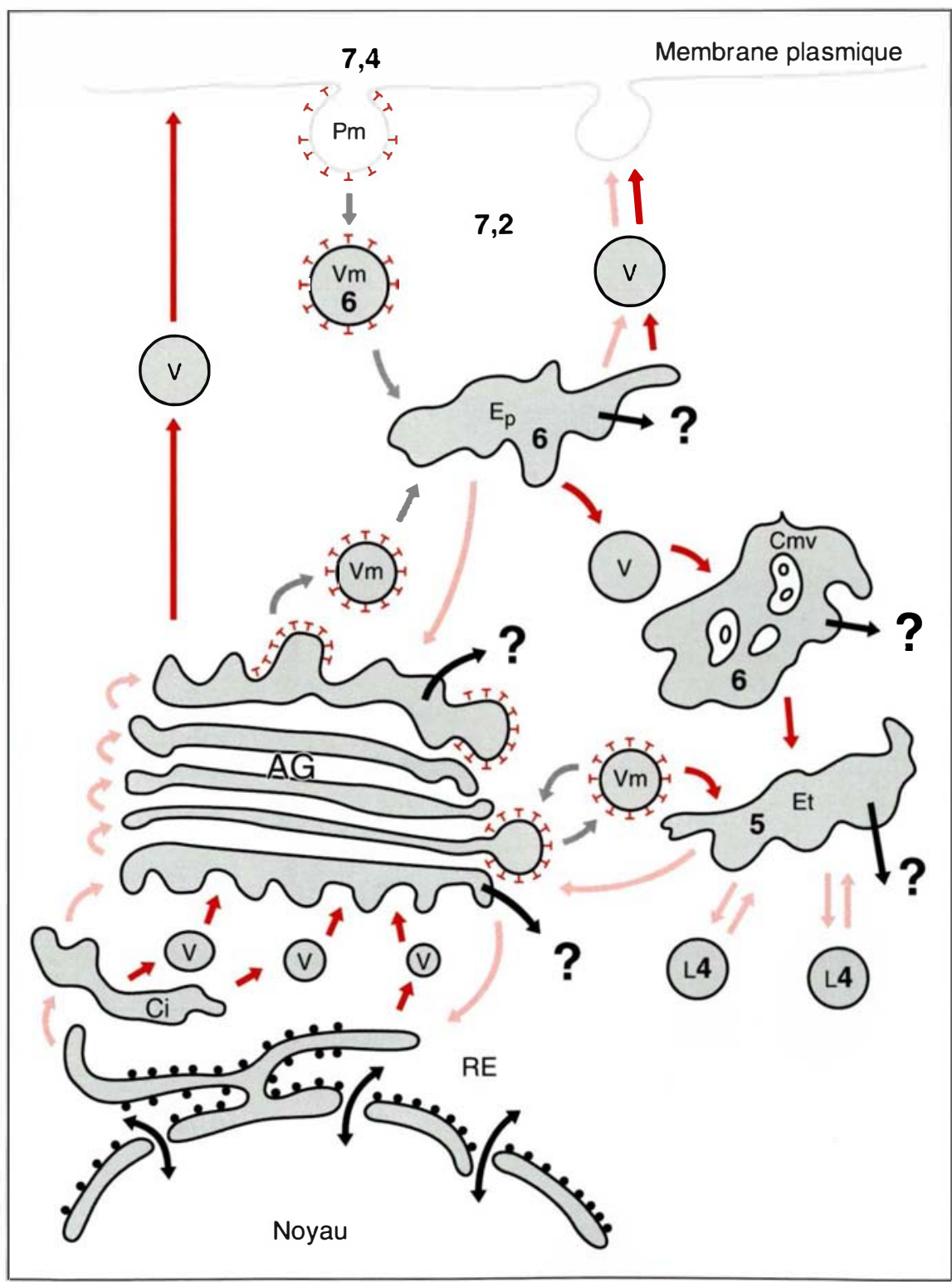




\section{Complexe entre un plasmide et un polycation lié à l'insuline}

La sérum albumine est une protéine acide, peu soluble en milieu acide, contrairement aux globulines; en électrophorèse, elle est beaucoup plus cathodique que les plus acides des globulines, les globulines $\alpha$. La sérum albumine peut, comme toutes les protéines, être transformée en un dérivé basique par substitution de ses groupements carboxyliques par un réactif cationique (le chlorure de N-éthyl-N'diméthylamino propyl carbodiimide $\left[\mathrm{C}_{2} \mathrm{H}_{5}-\mathrm{N}=\mathrm{C}=\mathrm{N}-\left(\mathrm{CH}_{2}\right)_{3}-\stackrel{+}{\mathrm{N}} \mathrm{H}\left(\mathrm{CH}_{3}\right)_{2}\right.$ [22]]). La sérum albumine ainsi rendue plus cationique est accolée à l'insuline après traitement au glutaraldéhyde. La conjugué est alors utilisé pour former un complexe avec un plasmide qui est efficace pour transfecter les hépatomes HepG2.

L'insuline a ćté également liée par pont disulfure à de la poly-L-lysine et le conjugué utilisé pour former un complexe avec un plasmide comportant le gène de l'antigène grand $T$ du
SV40. Après 2 heures à $37^{\circ} \mathrm{C}$, les cellules (hépatome humain) exprimant le récepteur de l'insuline ont incorporé le complexe qui se retrouve près du noyau et dans le noyau [23].

\section{Complexe entre un plasmide et un polycation lié à la transferrine}

Considérant que les cellules qui se multiplient ont besoin de fer, et donc expriment le récepteur de la transferrine, et que la transferrine entre dans la cellule sans être significativement transférée dans les lysosomes, la transferrine constitue un bon transporteur potentiel. Cependant, la transferrine ne donne pas de complexe stable avec l'ADN. En revanche, la transferrine liée à une protéine basique telle que la protamine ou la polylysine donne des complexes stables avec de l'ADN en double brin, de l'ADN en simple brin et même de l'ARN [24]. Un plasmide contenant le gène de la luciférase sous la dépendance du promoteur du virus du sarcome de Rous a été complexé à un conjugué transferrine-polylysine

Figure 2. Schéma du trafic intracellulaire de molécules telles que les protéines et les glycoprotéines. Pm : puits mantelé lrecouvert d'adaptine et de clathrine) ; $V m$ : vésicule mantelée ; $V$ : vésicule lisse ; $E p$ : endosome précoce : $C m v$ : corps multivésiculaire; Et : endosome tardif; $L$ : lysosome ; Ci : corps intermédiaire aussi appelé compartiment intermédiaire entre le réticulum endoplasmique et l'appareil de Golgi AG; RE : réticulum endoplasmique. Les flèches rouges correspondent à un transfert par des vésicules lisses, les flèches grises par des vésicules mantelées, les flèches roses par des vésicules ou des prolongements tubulaires transitoires, les flèches noires sont les chemins empruntés par les molécules libres, le point d'interrogation précise que le chemin est hypothétique. La transferrine suit un chemin limité $: P m \rightarrow V m \rightarrow E p$ $\rightarrow V \rightarrow$ membrane plasmique. La transferrine est associée à son récepteur pendant son voyage intracellulaire. L'asialoorosomucoïde suit un chemin plus complexe ; il est en effet transporté jusqu'aux lysosomes $L: P m, V m, E p \rightarrow C m v$ $\rightarrow E t \rightarrow L$. L'asialoorosomucoïde reste associé à son récepteur jusqu'à l'endosome précoce, il se dissocie en effet de son récepteur parce que l'environnement devient acide. Le récepteur est recyclé vers la membrane plasmique, pendant que l'asialoorosomucoïde est convoyé vers les endosomes tardifs et finalement dans les lysosomes. Une partie des protéines ou glycoprotéines intégrées par endocytose peuvent rejoindre l'appareil de Golgi et même le réticulum endoplasmique. Les valeurs numériques correspondent au $\mathrm{pH}$ des compartiments : 7,4 pour le compartiment extracellulaire, 7,2 pour le cytosol, aux environs de 6 dans les vésicules mantelées, les endosomes primaires et le corps multivésiculaire, aux environs de 5 dans les endosomes tardifs, et enfin aux environs de 4,5 dans les lysosomes. La chloroquine retarde l'acidification des divers compartiments, inhibe diverses hydrolases, et en particulier des protéases, et prévient les fusions entre vésicules et organites de la voie endosomique.

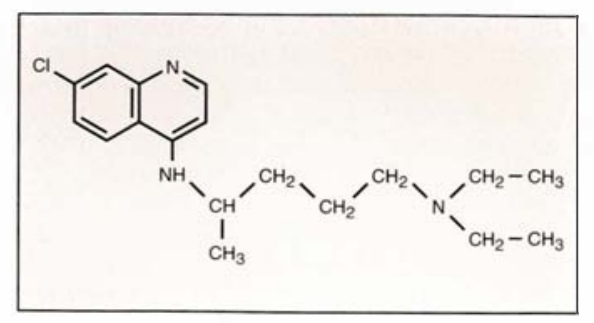

Figure 3. Structure de la chloro-

quine.

pour transfecter des cellules exprimant abondamment le récepteur de la transferrine.

Ce mode de transfection, qui est spécifique puisqu'il dépend de la présence du récepteur de la transferrine, est beaucoup moins efficace que la transfection utilisant le DEAE dextran. Cependant, lorsque la transfection est effectuée en présence de chloroquine (200 $\mu \mathrm{M})$, l'activité de la luciférase se rapproche de celle obtenue avec des cellules transfectées par la méthode au DEAE dextran [25]. Il est à noter que la chloroquine, quelle que soit sa concentration, n'améliore pas l'efficacité de la transfection obtenue par la méthode au DEAE dextran. L'efficacité de la transfection dépend de la densité des récepteurs de la transferrine présente à la surface des cellules, d'une part, et de la présence de chloroquine, qui retarde la dégradation du complexe, d'autre part. Lorsque l'incubation des cellules est faite en présence du complexe plasmide/transferrine-polylysine et de monensine, l'efficacité de la transfection est très faible, et cela qu'il y ait ou non de la chloroquine [26]. Ce résultat est probablement lié au fait qu'en présence de monensine, tous les compartiments cellulaires sont au même $\mathrm{pH}$, et que les processus de fusion membranaire naturels sont pratiquement inexistants.

L'incubation des cellules avec le complexe plasmide/transferrine-polylysine et en présence d'adénovirus défectifs ou inactivés $\left(5.10^{11} \mathrm{particules} / \mathrm{ml}\right)$ conduit à une transfection plus efficace qu'en présence de chloroquine $(100 \mu \mathrm{M})$, juqu'à 100 fois plus efficace [27]. Cette technique est également valable lorsque le plasmide est très gros (48 000 paires de bases). 


\section{RÉFÉRENCES}

28. Wagner E, Plank C, Zatloukal K, Cotten $\mathrm{M}$, Birnstiel ML. Influenza virus hemagglutinin HA-2N-terminal fusogenic peptides augment gene transfer by transferrin-polylysine-DNA complexes : toward a synthetic virus-like gene-transfer vehicle. Proc Natl Acad Sci USA 1992 ; 89 : 7934-8.

29. Derrien D, Midoux P, Petit C, et al. Muramyl dipcptide bound to poly-L-lysine substituted with mannose and gluconoyl residues as macrophage activators. Glycoconjugate J $1989 ; 6: 241-55$

30. Midoux $\mathrm{P}$, Nègre $\mathrm{E}$, Roche AC, et al. Drug targeting : anti-HSV-1 activity of mannosylated polymer-bound 9-(2-phosphonylmethoxyethyl) adenine. Biochem Biophys Res Commun 1990 ; 167 : 1044-9.

31. Nègre E, Chance ML, Hanboula SY, et al. Antilcishmanial drug targeting through glycosylated polymers specifically internalized by macrophage membrane lectins. Antimicrob Agents Chemother $1992 ; 36$ : 2228-32.

32. Midoux P, Mendes C, Legrand A, Raimond $J$, Mayer R, Monsigny $M$, Roche AC. Specific gene transfer mediated by lactosylated poly-L-lysine into hepatoma cells. Nucleic Acids Res 1993; 21 : 871-8.

33. White JM. Membranc fusion. Science $1992 ; 258$ : 917-24.

34. Takahashi S. Conformation of membrane fusion-active 20-residuc peptides with or without lipid bilayers. Implication of alpha-helix formation for membrane fusion. Biochemistry $1990 ; 29: 6257-64$.

35. Rafalski M, Artiz A, Rockwcll A. Membranc fusion activity of the influenza virus hemagglutinin : interaction of $\mathrm{HA} 2 \mathrm{~N}$ terminal peptides with phospholipid vesicles. Biochemistry 1991; 30 : 10211-20.

36. Murata M, Sugahara T, Takahashi S, Ohnishi SI. pH-dependent membrane fusion activity of a synthetic twenty amino acid peptide with the same sequence as that of the hydrophobic segment of influenza virus hemagglutinin. $J$ Biochem $1987 ; 102$ : 957-62.

37. Wagner E, Cotten M, Foisner R, Birnstiel ML. Transferrin-polylysine-DNA complexes : the effect of polycations on the structure of the complex and DNA delivery to cells. Proc Natl Acad Sci USA 1991; 88 :
Lorsque les cellules sont incubées avec le complexe plasmide/transferrine-polylysine en présence d'un peptide fusiogène, lui aussi lié à la polylysine, la transfection est plus cfficace qu'en présence de chloroquine ; lorsque l'incubation a lieu en présence de la chloroquine et du peptide fusiogène lié à la polylysine, la transfection est encore plus efficace [28].

\section{Complexe entre un plasmide et une polylysine glycosylée}

La polylysine glycosylée a été utilisée pour transporter avec efficacité divers agents thérapeutiques tels qu'un activateur de macrophages [29] ou des analogues de nucléotides à activité antivirale [30] ou antiparasitaire [31]. La spécificité cellulaire est conférée par la nature de la copule glucidique portée par la polylysine. Pour le transport des petites molécules, il convient de neutraliser les groupes aminés pour que le conjugué soit neutre, par acylation à l'aide de groupement gluconoyle, par exemple. En revanche, pour transporter de l'ADN, il faut conserver, au moins partiellement, les fonctions amines libres pour ne pas perdre le caractère polycationique des conjugués, caractère indispensable à la formation du complexe ADN/polylysine.

La glycosylation de la polylysine peut se faire soit en milieu aqueux, soit en milieu organique, puisqu'il ne s'agit pas d'une protéine mais d'un polymère. La substitution en milieu organique présente l'avantage de permettre l'utilisation d'une large gamme de réactifs, c'est-à-dire des réactifs utilisables en milieu aqueux ainsi que de nombreux autres qui sont insuffisamment stables en milieu aqueux; elle présente également l'avantage de permettre un meilleur contrôle des réactions et ainsi de maîtriser le degré de substitution. La présence de quelque 50 unités osidiques sur une molécule de polylysine contenant environ 190 résidus de lysine, confere au conju-

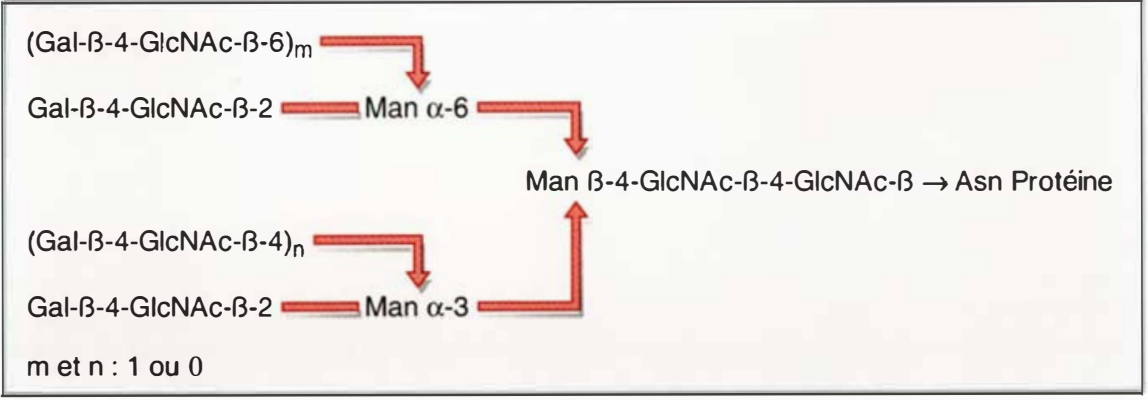

Figure 4. Structure des cinq oligosaccharides liés à une asparagine dans I'asialoorosomucoïde $(\mathbf{m}=0$ ou $1, n=0$ ou 1). La lectine membranaire des cellules du parenchyme hépatique reconnaît de façon préférentielle les structures dans lesquelles $\mathrm{m}$ et/ou $\mathrm{n}$ sont égaux à 1, c'est-à-dire les structures avec trois ou quatre galactoses en position terminale. Gal : galactose ; Man : mannose; GlcNac : N-acétylglucosamine; Asn : asparagine.

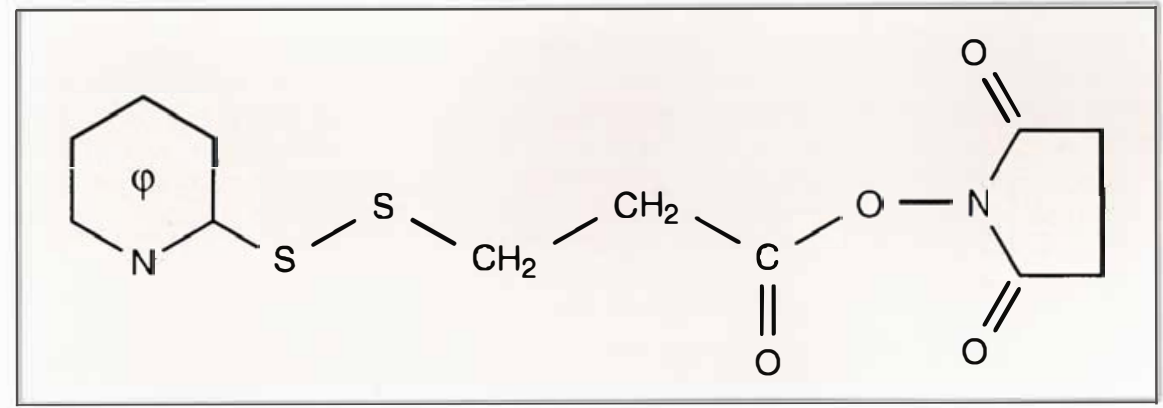

Figure 5a. Ester de l'acide pyridinyldithiopropanoïque et de I'hydroxysuccinimide (SPDP). 


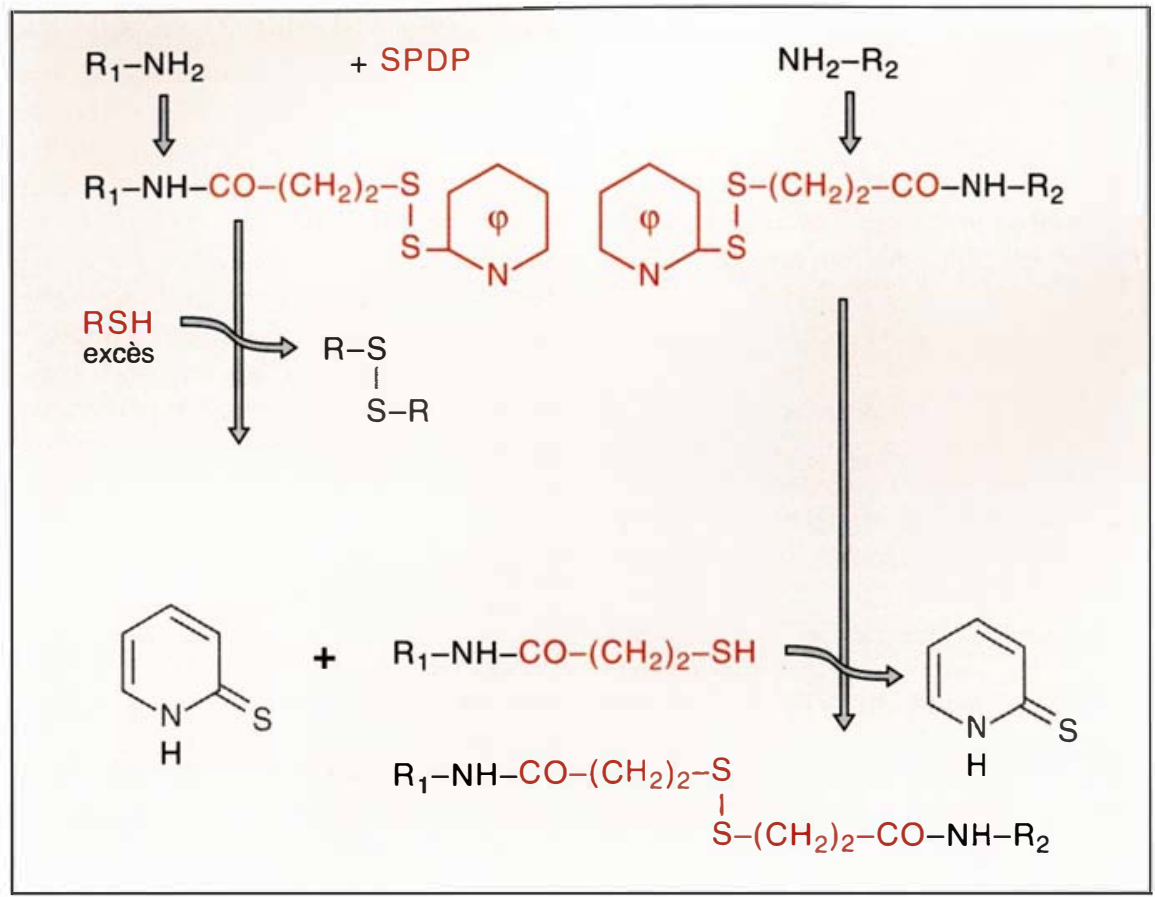

Figure 5b. Schéma réactionnel de la préparation des conjugués protéinepolylysine. $R_{1}$ : polylysine; $R_{2}$ : protéine ; $R-S H$ : agent réducteur.

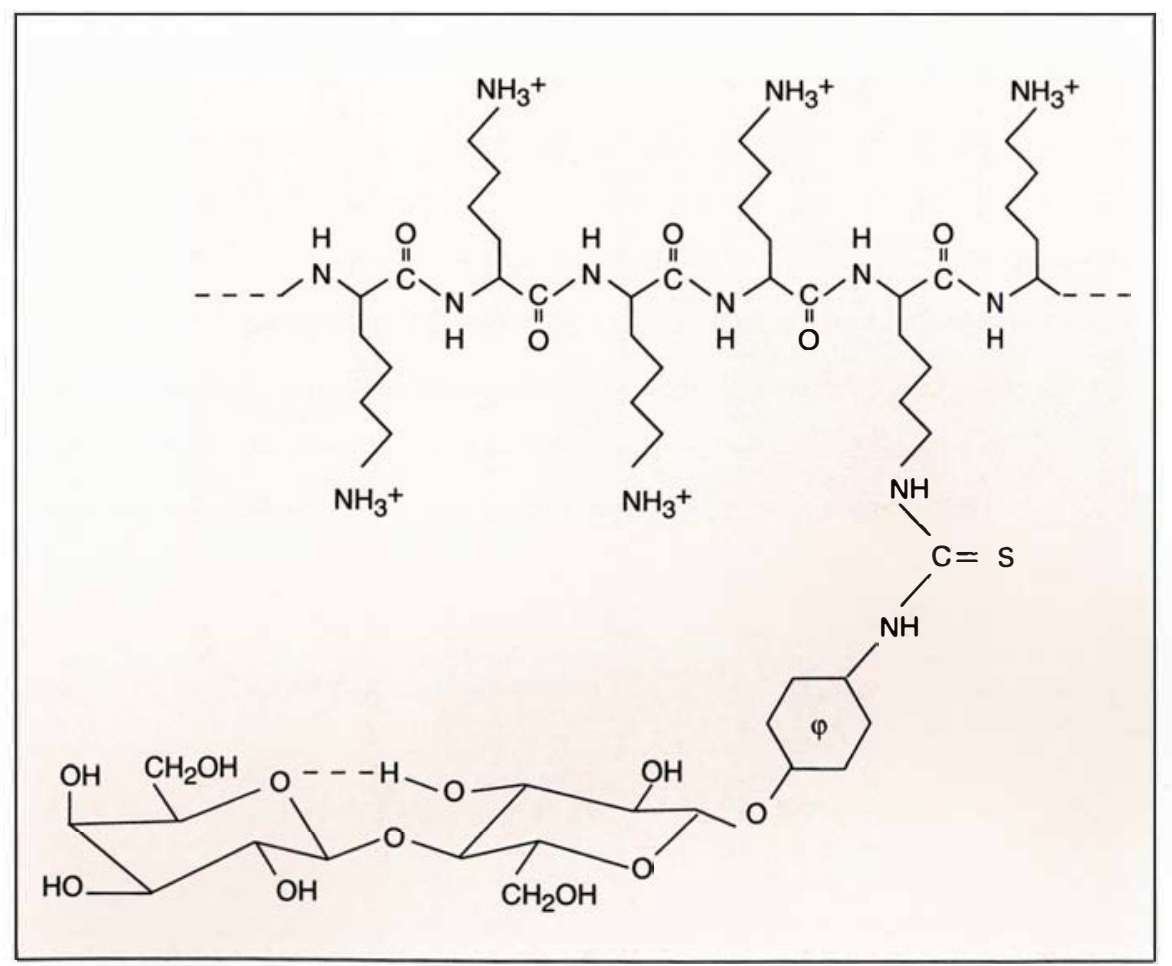

Figure 6. Schéma de la structure d'un fragment de polylysine substitué par des résidus de lactose (Gal- $\beta$-4-GIc- $\beta$-oxy-4-phénylthiocarbamyl-1).

$\mathrm{m} / \mathrm{s} n^{\circ} 4$ vol. 9, auril 93 gué, par un phénomène analogue à l'avidité, la capacité d'être reconnu de façon efficace par les lectines membranaires ayant une affinité, même faible, pour l'unité osidique liée à la polylysine.

La spécificité des polylysines glycosylées est liée à l'unité osidique portée par la polylysine [32]. Par exemple, une polylysine lactosylée ( $\mathrm{Gal}-\beta$ Glc) (figure 6) est reconnue par des hépatocytes et des cellules de lignées telles que HepG2 qui expriment la lectine membranaire spécifique des glycoconjugués possédant du galactose non substitué en position externe comme dans le cas des asialoglycoprotéines. En revanche, cette polylysine lactosylée n'est pas reconnue par des cellules qui n'expriment pas cette lectine, telles les cellules tumorales humaines HeLa. La polylysine mannosylée n'est, quant à elle, reconnue ni par les cellules du parenchyme hépatique, ni par les cellules HepG2 qui n'expriment pas la lectine membranaire spécifique du mannose, et le complexe plasmide-polylysine mannosylée est, dans ces conditions, inefficace.

Les polylysines glycosylées sont très solubles en milieu aqueux, dans du sérum physiologique par exemple. La formation du complexe plasmidepolylysine glycosylée est immédiate par simple mélange des deux constituants : le plasmide d'une part, la polylysine glycosylée d'autre part, dissous dans du milieu de culture (figure 7). Le complexe en solution est alors prêt à l'emploi. Les caractéristiques physicochimiques de la polylysine glycosylée en font un outil beaucoup plus facile à utiliser que les conjugués protéine-polylysine. Lorsque le complexe est mis en présence de cellules possédant une lectine membranaire ayant de l'affinité pour l'unité osidique présente sur la polylysine, il est rapidement internalisé par endocytose et se retrouve dans des vésicules du compartiment endocytique. Pour que le transfert de gène soit efficace, il faut ajouter un agent favorisant cinétiquement ou physiquement le passage à travers la membrane dans le cytosol. La chloroquine est efficace lorsqu'elle est ajoutée en même temps ou légèrement après le complexe plasmide-polylysine glycosylée. La concentration en chloroquine 
utilisée $(100 \mu \mathrm{M})$ est approximativement celle que l'on trouve dans les tissus des personnes qui utilisent la chloroquine pour prévenir le développement du paludisme.

L'utilisation des peptides fusiogènes comme agent facilitant physiquement le passage transmembranaire du gène transféré dérive de l'observation que les orthomyxovirus, comme celui de la grippe, ont un peptide $\mathrm{N}$-terminal qui est fusiogène en milieu acide mais inactif en milieu neutre [33].

Un eicosapeptide ayant la séquence du peptide $\mathrm{N}$-terminal de la protéine de fusion du virus de la grippe (hémagglutinine) a été synthétisé, et ses propriétés ont été étudiées et comparées avec celles de peptides de même longueur mais de séquence légèrement différente. Les peptides possédant des résidus glutamyl en position $n+4$ (ou $n+3)$ et des acides aminés hydrophobes entre eux sont solubles en milieu neutre ou alcalin, et ont une structure désordonnée. En milieu légèrement acide, les peptides prennent une conformation ordonnée (figure 8) et, en présence de vésicules lipidiques, ils prennent nettement une structure hélicoï-

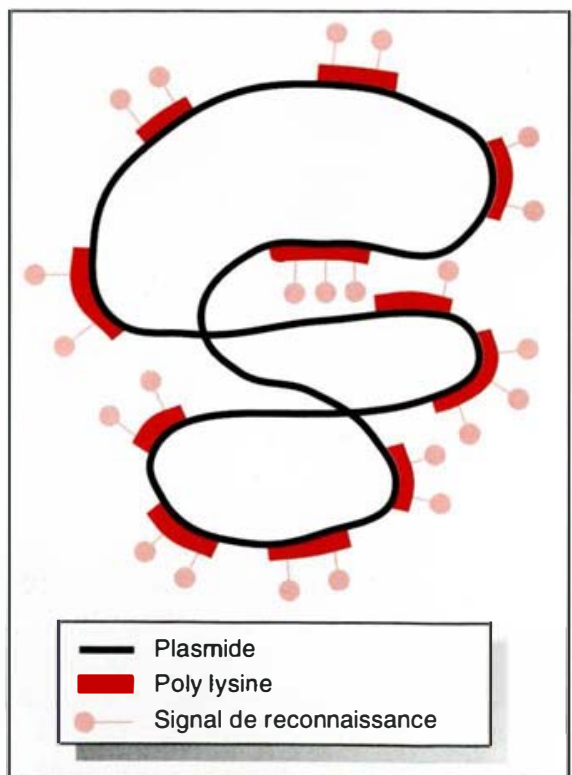

Figure 7. Schéma du complexe ionique entre le plasmide (ADN double brin) polyanionique et la polylysine (substituée par un signal dale $\alpha$ [34] et induisent la fusion entre vésicules $[35,36]$.

L'efficacité de transfert de gènes avec un plasmide transporté par une polylysine lactosylée est fortement augmentée lorsque l'incubation des cellules est effectuée en présence d'un tel peptide (5 à $10 \mu \mathrm{M}$ ) :

\section{GLFEAIAEFIEGGWEGLIEGCA.}

L'activité de l'enzyme exprimée, la luciférase dans ce cas, est 500 fois plus élevéc qu'en absence de peptides fusiogènes et neuf fois plus élcvée qu'en présence de chloroquine [32].

Récemment, Wagner et al. [27] ont utilisé un autre peptide ayant une séquence voisine de celle du peptide $\mathrm{N}$-terminal de la même protéine :

\section{GLFEAIAGFIENGWEGMIDGGGC.}

Ce peptide n'a pas été utilisé à l'état libre mais lié par un pont disulfure à de la polylysine. Un complexe ternaire a été préparé par mélange d'une solution de plasmide, d'une solution du conjugué transferrinepolylysine et enfin d'une solution du conjugué peptide fusiogène-polylysine. L'activité de l'enzyme exprimée après transfection dépend fortement des rapports et des concentrations des conjugués de la polylysine. Cette activité est encore augmentée lorsque les cellules sont incubées avec le même complexe ternaire en présence de chloroquine pendant 24 heures. Cc résultat surprenant pourrait être expliqué par une inhibition prolongée des processus de transfert aux lysosomes et par une meilleure protection contre la dégradation par les hydrolases.

\section{Conclusions et perspectives}

La polylysine, un polycation, interagit spontanément avec un acide nucléique pour donner un complexe suffisamment stable pour pouvoir être isolé et caractérisé. Le plasmide au contact du polycation se condense en une pelote [37], ce qui facilite probablement la pénétration dans les cellules. Cette pénétration est fortement augmentée lorsque la polylysine est substituée, au moins partiellement, par une protéine telle qu'une asialoglycoprotéine, la transferrine ou l'insuline, ou par des petits signaux

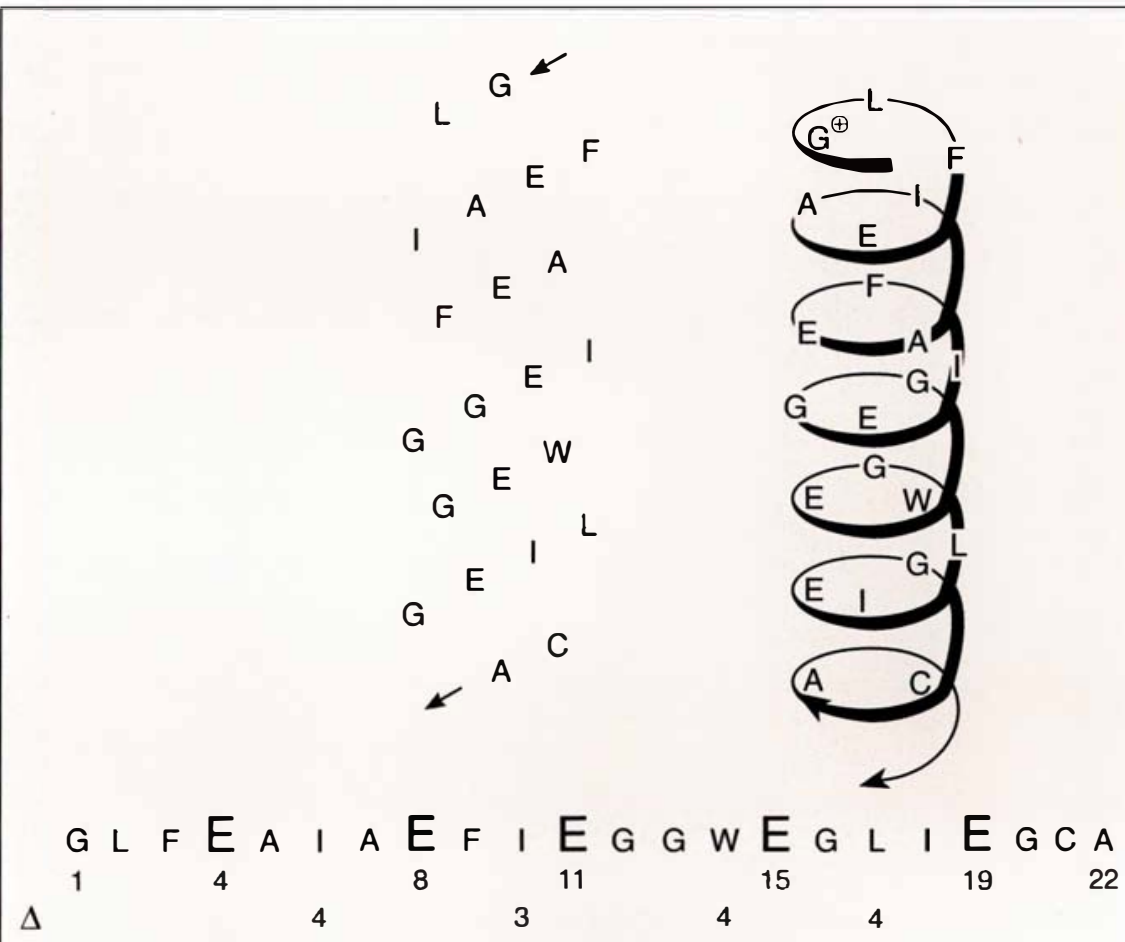

Figure 8. Disposition des acides aminés dans un peptide fusiogène contenant cinq résidus acide glutamique en position $n+\Delta(\Delta=3$ ou 4) en milieu légèrement acide. 
de reconnaissance comme des osides. Dans tous les cas évoqués ci-dessus, les ligands (protéiques ou osidiques) sont reconnus par des récepteurs présents à la surface des cellules, et ces récepteurs induisent activement l'internalisation de leur ligand. Pour que la transfection soit efficace, il faut cependant une aide, qui est apportée soit par une amine telle que la chloroquine, qui ralentit le transfert vers les lysosomes et la dégradation du matériel endocyté, soit par un peptide fusiogène, qui déstabilise la membrane des endosomes lorsque l'environnement est suffisamment acide. Le peptide fusiogène peut être libre ou lié à un transporteur.

L'efficacité des systèmes polylysinesignal de reconnaissance est remarquable pour la transfection transitoire de cellules en culture. Elle est spécifique dans la mesure où l'efficacité dépend de la présence du récepteur membranaire correspondant au signal de reconnaissance porté par la polylysine.

Ce système peut être utilisé également pour obtenir une transfection stable. Les premiers résultats (A. Legrand et P. Midoux) avec des cellules HOS (lignée d'un ostéosarcome humain) montrent que le nombre de colonies est au moins dix fois plus élevé que celui obtenu par une transfection au phosphate de calcium.

Les premières expériences de transfection in vivo par le conjugué asialoglycoprotéine polylysine montrent qu'à condition de réaliser, immédiatement après l'injection du complexe plasmide transporteur, une hépatectomie partielle, la protéine transfectée est expriméc pendant plusieurs semaines [19-21].

Les conditions optimales d'obtention de transfection stable n'ont pas encore été précisées ; on peut supposer que de nouveaux progrès seront réalisés dans ce domaine dans un proche avenir. Le système polylysinesignal de reconnaissance - en tant que transporteur de plasmide pour réaliser des transfections stables de façon sélective vis-à-vis de cellules cibles choisies préalablement - peut être élargi à des signaux de reconnaissance autres que ceux décrits jusqu'à présent. En ce qui concerne les signaux osidiques, il est possible $\mathrm{m} / \mathrm{s} n^{\circ} 4$ vol. 9, auril 93 d'envisager la transfection sélective de nombreux types cellulaires. On sait en effet que les cellules endothéliales, divers leucocytes, les monocytes, les macrophages, certains lymphocytes, etc., expriment à leur surface des lectines reconnaissant sélectivement des structures oligosaccharidiques complexes (oligomannose, Lewis ${ }^{x}$, sialyl Lewis ${ }^{x}$, etc.) [2-4]. Il est aujourd'hui possible de préparer, à partir de composés naturels ou par synthèse, de telles molécules. Très rapidement, il sera possible de transfecter de façon sélective les cellules exprimant des lectines reconnaissant très efficacement les complexes plasmide-polylysine-oligosaccharide. De façon analogue, des polylysines substituées par des peptides reconnus par des récepteurs membranaires permettront de diriger vers les cellules exprimant ces récepteurs le complexe plasmide-polylysine. De façon très générale, on peut donc prévoir que, dans l'avenir, fleuriront des polylysines substituées par des ligands de faible masse moléculaire, spécifiques de récepteurs membranaires de cellules différenciées et probablement aussi de cellules souches. Alors, ce système pourra devenir un précieux outil dans le développement de la thérapie génique

\section{Remerciements}

Les travaux du laboratoire dans ce domaine ont bénéficié du concours de P. Bouchard, M.-T. Bousser, M. Dubois, A. Legrand, E. Martin, C. Mendès, S. Nuques, R. Mayer et J. Raimond.

\section{TIRÉS A PART}

M. Monsigny.

\section{Summary}

Targeted plasmid-polylysine complexes as putative tools to selectively transfer genes $e x$ vivo and in vivo

Gene therapy will be an ideal method when it is possible to transfer DNA in cells with a great efficacy and an absolute safety in vivo. Along with viral carriers which are efficient but may not be strictly safe, non viral carriers could be advantageously used. Polylysine, a polycation giving stable DNA complexes, is proposed as the basis of such a carrier system. Indeed, polylysine substituted with either a protein easily taken up by cells due to specific cell surface receptors, or carbohydrate moieties which selectively interact with lectins expressed at the surface of specific cells, allows a cell specific delivery of genes. In addition, when such a targeted carrier is used together with components that protect the plasmidtargeted polylysine complex from the hydrolytic activities of lysosomes and/or help the complex to cross the cell membranes to reach the cytosol, the specificity and the efficacy of the transfection are conspicuously higher.

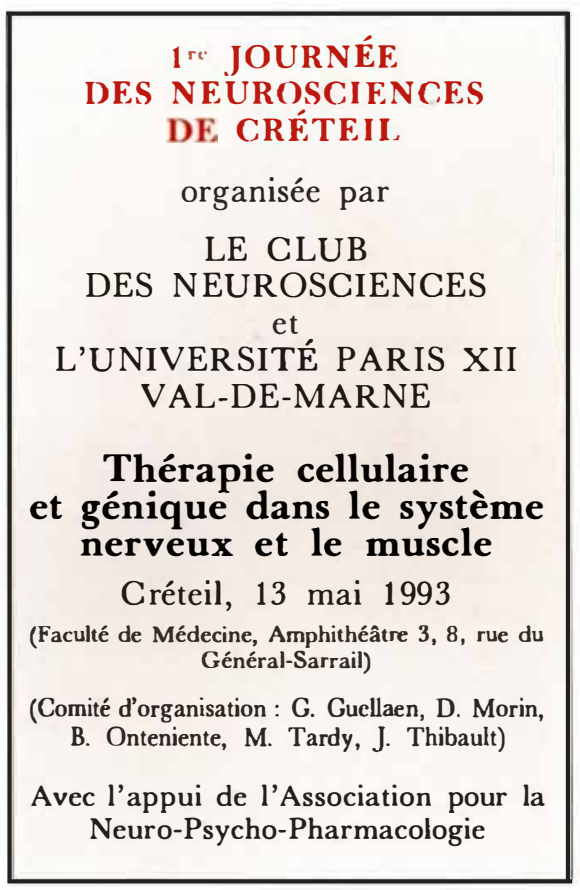

